\title{
Protest gegen die Verpflichtung zur Übernahme des Amtes des Exegeten
}

Dieter Hagedorn (Heidelberg) und Günter Poethke (Berlin)

P.Ien. Inv. 60A recto

$16 \times 10,5 \mathrm{~cm}$

2. Hälfte 3. Jh. n.Chr.

Das mittelbraune Papyrusblatt ist links und unten abgebrochen. Links fehlen vom Text 3-4 Buchstaben, ab Z. 7 etwa 10-11 Buchstaben je Zeile. Oben ist ein Freirand von $1,5-2 \mathrm{~cm}$ Höhe erhalten. Die Schrift in schwarzer Tinte verläuft parallel zur Faser. Die letzten Buchstaben der Zeile reichen, durch ausgedehnte Ausläufer verlängert, oft bis zum Rand. Insgesamt gewinnt man den Eindruck einer durch den Kanzleistil heeinflußten Schrift. Eine Datierung ist nicht erhalten, aber die Ehrentitel der Stadt Hermupolis in Z. 4 weisen in die Zeit zwischen 267 und $306 \mathrm{n}$.Chr. (s. den Kommentar), was durchaus auch zum Schriftbild paßt. Auf dem Verso befindet sich, ebenfalls parallel zur Faser geschrieben, die Aufstellung über die Ausgabe von Wein und Weintrauben SB VI 9132.1

Aurelios Kopreas, S. des Melas, war von drei amtierenden Exegeten von Hermupolis, nämlich Aur. Hermammon, S. des Triadelphos, Aur. -mmon, S. des Hermophilos, und Aur. Hierax, S. des Hermes als Nachfolger des Erstgenannten im Amte des Exegeten benannt worden, wobei die beiden Letztgenannten als

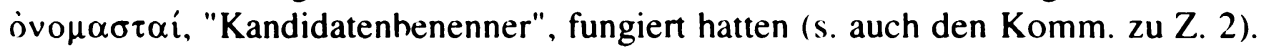
Am Vortage hatte Aurelios Kopreas durch einen Aushang von diesem Vorgang Kenntnis erhalten; jetzt richtet er mit der Begründung, seine finanziellen Mittel seien zur Übernahme des Amtes völlig ungenügend, den vorliegenden Protest

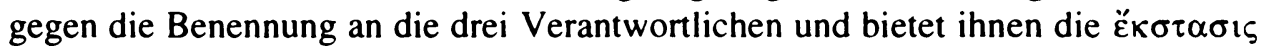
(cessio bonorum) an, d.h. den Verzicht auf sein gesamtes Vermögen zu ihren Gunsten, unter der Bedingung, da $ß$ sie sich daraufhin bereiterklären, an seiner Stelle das Amt zu übernehmen.

Es sind uns aus dem 3. Jh. n.Chr. auch andere Texte vergleichbaren Inhalts erhalten, die beredt Zeugnis davon ablegen, wie prekär die finanzielle Situation der für derartige Liturgien und speziell für die munizipalen Ämter in Frage kommenden Bevollkerungsschicht der Metropolen in ebendieser Zeit geworden war. Vgl. zuletzt P.Hamb. IV 268 (Oxyrh.; 289 n.Chr.), wo in der Einleitung die Parallelen und die wichtigste Literatur genannt sind.

${ }^{1}$ Ed. pr.: F. Zucker, Papyri lenenses, Aegyptus 32 (1952) 73-84. hier 80-84. 


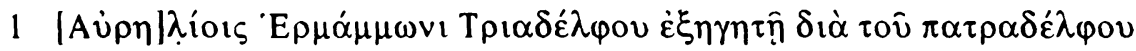

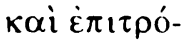

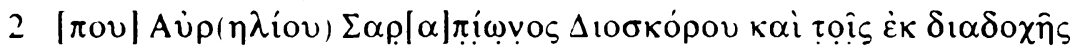
$\dot{o v} 0 \mu \alpha \sigma \tau \hat{\varepsilon} \varsigma \sigma o v$

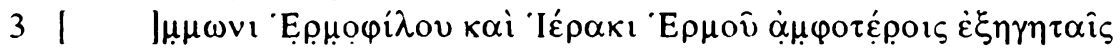

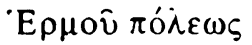

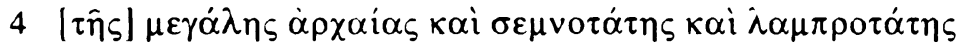

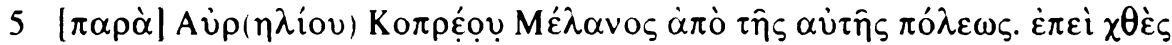
$\ddot{\eta} \tau \iota \varsigma \hat{\eta} v \overline{i \gamma}$.

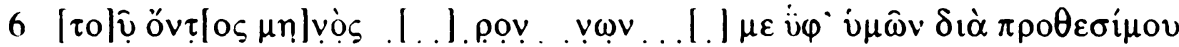

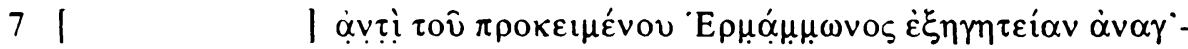

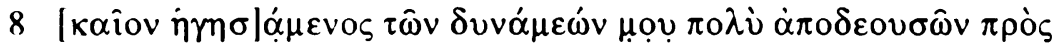

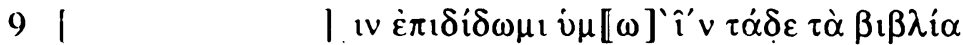
$\dot{\varepsilon} \xi_{1 \sigma \tau \alpha \nu o ́ \mu \varepsilon v o s}$

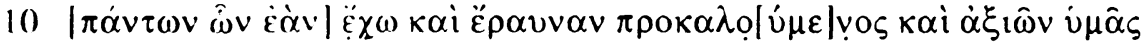
111

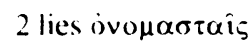

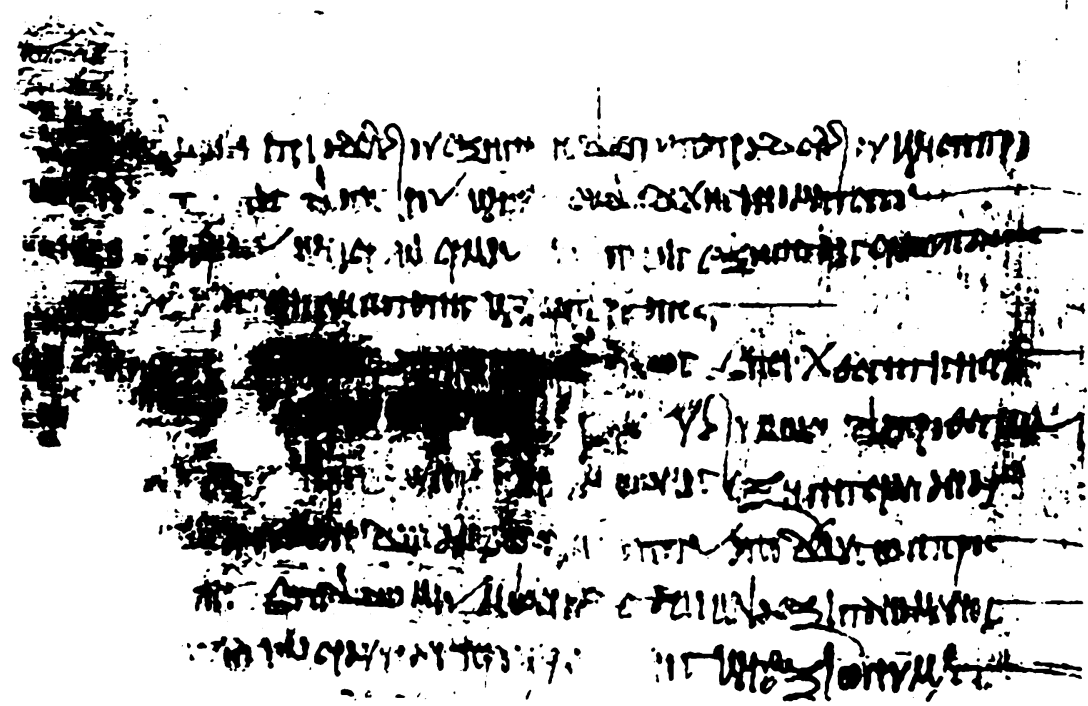

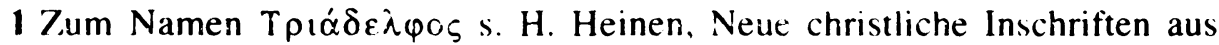
Hermonthis, ZPE 81 (1990) 270-274, bes. 270-273; J. Quaegebeur, Greco-

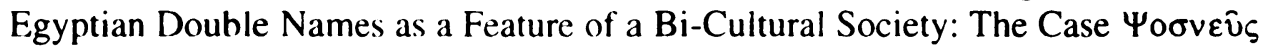

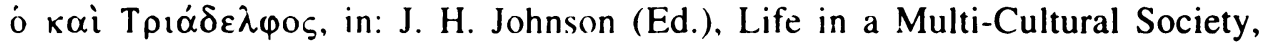
Chicago 1992. S. 265-272. 


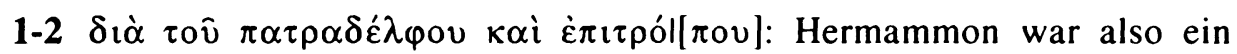
Waisenkind und wurde als Minderjähriger unter 14 Jahren von einem tutor

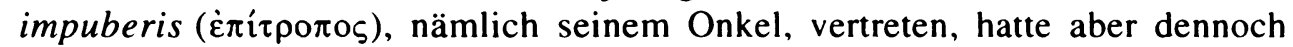
bereits das Amt des Exegeten übernommen. Zur Unterscheidung zwischen

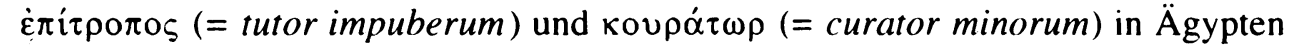
nach der Constitutio Antoniniana s. A. Arjava, Die römische Vormundschaft und das Volljährigkeitsalter in Ägypten, ZPE 126 (1999) 202-204.

Es ist auffällig, daß von den 11 Belegen für das Wort $\pi \alpha \tau \rho \alpha ́ \delta \varepsilon \lambda \varphi \circ \varsigma$, welche die Suche in der DDBDP erbringt, 7 aus Hermupolis stammen.

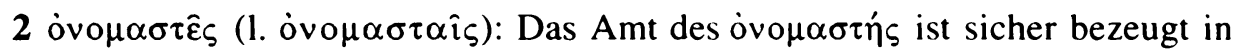

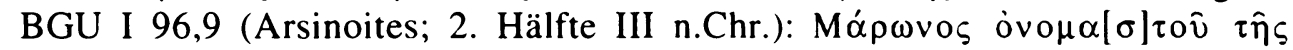
$\delta \varepsilon \kappa \alpha \pi \rho \omega \tau i \alpha \varsigma$ und P.Ryl. II 302 descr., Z. 14 (Herkunft unbekannt; 3. Jh. n.Chr.),

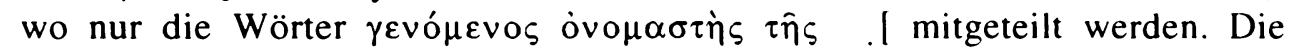
òvo $\mu \alpha \sigma \tau \alpha i ́$ waren ganz offenbar für die Benennung der Kandidaten für die Neubesetzung der munizipalen Ämter zuständig; daß amtierende Beamte (in unserem Fall also Exegeten) in dieser Weise für Amtsnachfolger zu sorgen hatten, ist gut bezeugt; s. speziell für die Exegeten z.B. P.Oxy. XII 1413 (Oxyrh.; 272 n.Chr.) und vgl. E.P. Wegener, The $\beta o v \lambda \dot{\eta}$ and the Nomination to the $\alpha \rho \chi \alpha \dot{i}$ in the $\mu \eta \tau \rho о \pi$ ó $\lambda \varepsilon 1 \varsigma$ of Roman Egypt, Mnemosyne, 4. Ser. 1 (1948) 15-42. 115-132. 297326 , bes. $19-42=$ S. 64-79 in dem Neudruck in P.Lugd. Bat. XXIII (1985) S. 62-114. Problematisch sind an unserer Stelle allerdings der Begriff $\dot{\varepsilon} \kappa$

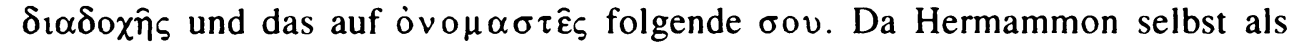
$\dot{\varepsilon} \xi \eta \gamma \eta \tau \dot{n} \varsigma$ und nicht als $\dot{\varepsilon} \xi \eta \gamma \eta \tau \varepsilon \dot{v} \sigma \alpha \varsigma$ bezeichnet wird, war er noch im Amt, so daß $\dot{\varepsilon} \kappa \delta 1 \alpha \delta \circ \chi \hat{\eta} \varsigma$ nicht "in deiner Nachfolge stehend" bedeuten kann. Alle drei in der Adresse genannten Personen sind amtierende Exegeten. Wir nehmen daher an,

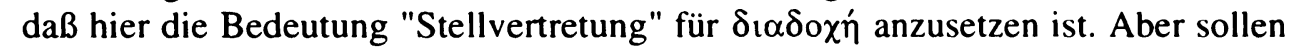

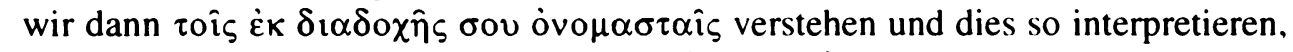

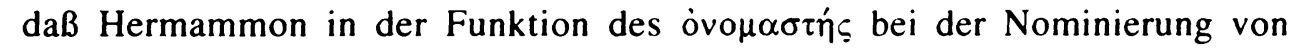
Kopreas durch zwei Kollegen vertreten worden ist? Eher ist unseres Erachtens der ganze Ausdruck mit $\dot{\varepsilon} \kappa$ mißraten, und wif sollen "als Kandidatenbenenner für deine Nachfolge" verstehen. Für diese Interpretation spricht jedenfalls ganz klar die Information von Z. 7, wo es heißt, daß es um einen Ersatz für Hermammon geht. Man könnte zuletzt auch daran denken, daß mit $\Sigma o v$ - bereits der Name des ersten Mannes beginnt, aber $\Sigma o v \chi \alpha ́ \alpha \mu \omega v$ - und dies käme zunächst in Betracht wäre ein Name, der eher in den Arsinoites weist, und es wäre auch nicht einzusehen, warum der Schreiber in diesem Fall nicht den vorhandenen Platz ausgenützt, sondern $\sigma o v$ mit einem so weit ausladenden $v$ geschrieben haben sollte. Wir scheiden daher diese Möglickeit aus.

3 Am Anfang der Zeile stand wohl im Dativ ein auf $-\alpha \dot{\alpha} \mu \omega v$ endender Name,

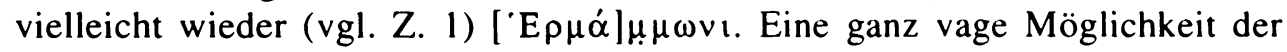
Identifizierung von 'Í́ $\rho \alpha \xi$ 'E $\rho \mu$ ô gibt es vielleicht in P.Vind. Bosw. 4,2 (Herm.;

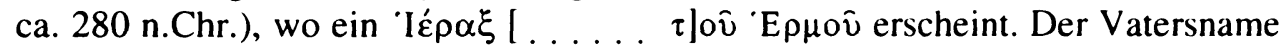
war offenbar kurz, und 'E $\rho \mu$ ô würde dem vorhandenen Platz wohl entsprechen. 


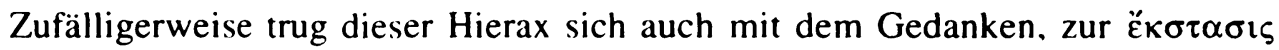
Zuflucht zu nehmen (s. Z. 3).

$4 \mathrm{~N}$. Litinas, Hermou polis of the Thebais. Some Corrections and Notes Concerning its Name and Epithets, APF 41 (1995) 66-84, bes. 81-82, hat beobachtet,

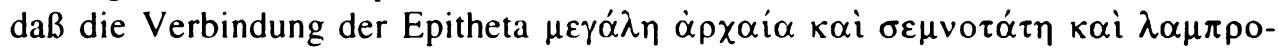
$\tau \alpha \dot{\tau} \tau$ bisher nur in der Zeit zwischen 267 und $306 \mathrm{n}$.Chr. bezeugt ist. In diesen Zeitraum würde man daher zunächst einmal auch den vorliegenden Text setzen.

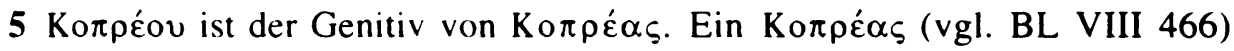

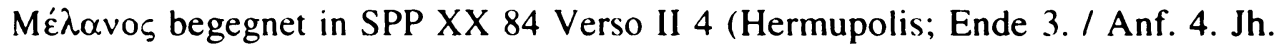
n.Chr.); er könnte durchaus mit dem vorliegenden identisch sein.

6-9 Wenngleich der generelle Sinn der Zeilen klar ist, haben wir doch keinen Vorschlag für die Rekonstruktion ihres Wortlauts anzubieten.

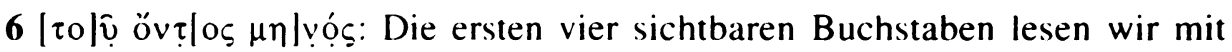
großer Zuversicht, so daß sich die vorgeschlagene Ergänzung anbietet. In Anbetracht der Schwierigkeiten danach sollte man aber darauf hinweisen, daß die Wendung $\chi \theta \dot{\varepsilon} \varsigma \ddot{\eta} \tau \iota \varsigma \hat{\eta} v \bar{i}$ nicht unbedingt einer Ergänzung bedarf; vgl. z.B.

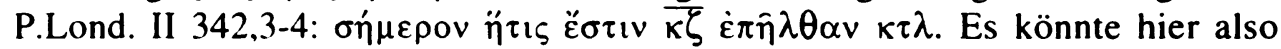
etwas gänzlich anderes gestanden haben. Danach - für eine namentliche Nennung des Monats scheint überhaupt kein Platz zu sein - erwartet man etwas wie "ich erfahren habe, da $B$ ich von euch benannt (bzw. vorgeschlagen, bestimmt) worden bin", aber die Tinte ist derartig verschmiert, da $\beta$ uns keine Lesung gelungen ist. Das $\mu \varepsilon$ nach der letzten Lücke ist unseres Erachtens das Subjekt in einem Acl.

$\pi \rho \circ \theta \varepsilon \sigma i \mu o v:$ Das Wort $\pi \rho \circ \theta \varepsilon \dot{\sigma} \mu_{\mu o v}$ ist bisher erst einmal in P.Panop. $29=$ SB XII 11222,19 belegt (noch nicht in LSJ, New Suppl. aufgenommen), wo die Bedeutung "für den Aushang bestimmte Kopie" erschlossen worden ist; vgl. den Kommentar zur Erstedition. Einfach "Aushang" wäre aber sicherlich ebenfalls zutreffend.

7 In der Lücke zu Beginn der Zeile könnte ein Infinitiv gestanden haben, der $\dot{\varepsilon} \xi \eta \gamma \eta \tau \varepsilon i \alpha v$ als Objekt bei sich hatte, also vielleicht mit der Bedeutung "zu

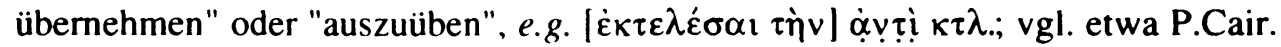

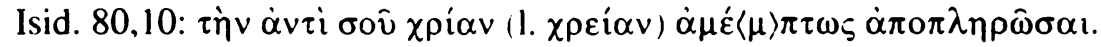

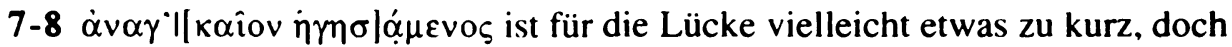
vgl. zu der Verbindung mit $\dot{\varepsilon} \pi \_\delta i \delta \omega \mu$ ı P.Oxy. VI 895,10-11 (305 n.Chr.) und (weitgehend ergänzt) BGU XII 2134,6.

$8 \mathrm{Zu}$ der Formulierung, mit der Kopreas darauf hinweist, da $\beta$ seine finanziellen Mittel zur Durchführung der Liturgie nicht ausreichen, vgl. P.Hamb. IV 268,7

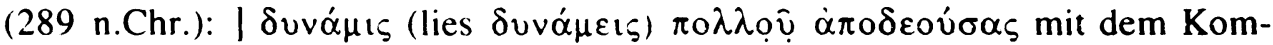
mentar.

9 I. $v$ : Vielleicht lọıv. Darin könnte man das Ende eines Substantivs im Akkusativ sehen, der von $\pi \rho$ ò $\varsigma$ in Z. 8 abhängt. 


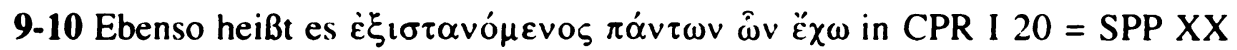
$54=$ W.Chr. 402.7 (250 n.Chr.).

10 Ëpauva hier in der Bedeutung "Untersuchung": vgl. P.Oxy. I $67=$ P.Lond. III 754 descr. = M.Chr. 56,18 (338 n.Chr.).

Zwischen $\kappa \alpha i$ und $\dot{\alpha} \xi_{\imath} \hat{\omega} v$ glaubt man ein hineingequetschtes $\tau$ zu erkennen; es handelt sich vielleicht um einen mißglückten ersten Ansatz zu dem Alpha, das dann beim zweiten Anlauf in anderer Gestalt ausgeführt wurde.

\section{Übersetzung}

An die Aurelier Hermammon, den Sohn des Triadelphos, Exegeten, vertreten durch den Vatersbruder und Vormund Aurelios Sarapion, den Sohn des Dioskoros, und die für deine Nachfolge als Kandidatenhenenner fungierenden -mmon. den Sohn des Hermophilos, und Hierax. den Sohn des Hermes, beide Exegeten der großen, alten, sehr angesehenen und glänzenden Stadt Hermupolis, von Aurelios Kopreas, dem Sohn des Melas, aus derselben Stadt.

Nachdem ich gestern, welches der 13. des gegenwärtigen Monats war, durch einen Aushang erfahren habe, daß ich von euch dazu bestimmt worden bin, anstelle des oben genannten Hermammon das Amt des Exegeten zu übernehmen, hielt ich es, da meine Mittel bei weitem nicht für dessen Übernahme ausreichen. für notwenig, euch dieses Gesuch einzureichen, indem ich auf alles, was ich besitze, verzichte. eine Untersuchung beantrage und euch auffordere --- 